\title{
Are digital picturings representations?
}

\author{
Annamaria Carusi \\ Oxford e-Research Centre \\ 7 Keble Road \\ Oxford OX1 3QG, UK \\ annamaria.carusi@oerc.ox.ac.uk
}

\author{
Gordana Novakovic \\ Artist-in-Residence \\ UCL Dept. of Computer Science \\ 40B Cavendish Road \\ London NW7 6XP, UK \\ gordana.novakovic@gmail.com
}

\author{
Timothy Webmoor \\ Institute for Science, Innovation \\ and Society \\ Park End Street \\ Oxford OX1 1HP, UK \\ timothy.webmoor@sbs.ox.ac.uk
}

\begin{abstract}
The philosopher of art Roger Scruton has claimed that photographic images are not representations, on the basis of the role of causal rather than intentional processes in arriving at the content of a photographic image (Scruton, 1981). His claim was controversial at the time, and still is, but had the merit of being a springboard for asking important questions about what kinds of representation result from the technologies used in depicting and visualising. In the context of computational picturing of different kinds, in imaging and other forms of visualisation, the question arises again, but this time in an even more interesting form, since these techniques are often hybrids of different principles and techniques. A digital image results from a complex interrelationship of physical, mathematical and technological principles, embedded within human and social situations. This paper consists of three sections, each presenting a view of the question whether digital imaging and digital visual artefacts generally are representations, from a different perspective. These perspectives are not representative, but aim only to accomplish what Scruton's paper did succeed in accomplishing, that is, being a provocation and a springboard for a broader discussion.
\end{abstract}

Digital images. Visualisation. Representation. Philosophy. Art. Science and technology studies.

\section{INTRODUCTION}

The philosopher of art Roger Scruton has claimed that photographic images are not representations, on the basis of the role of causal rather than intentional processes in arriving at the content of a photographic image (Scruton, 1981). His claim was controversial at the time, and still is, but had the merit of being a springboard for asking important questions about what kinds of representation result from the technologies used in depicting and visualising. In the context of computational picturing of different kinds, in imaging and other forms of visualisation, the question arises again, but this time in an even more interesting form, since these techniques are often hybrids of different principles and techniques. A digital image results from a complex inter-relationship of physical, mathematical and technological principles, embedded within human and social situations. An extremely common, even natural, assumption about digital images is that they, like other images, are representations. Despite its apparent naturalness, it is an assumption that can be questioned. If digital images, or indeed other visual artefacts, are representations, why - in what ways are they continuous with other activities and practices of representing, and the goals and purposes of other forms of representations? What do they represent? What are the specific forms and modes of representation of computational and digital methods, and in what ways are they modulating expectations of representation, generally and specifically, in art?

This paper speaks to the panel of the same title at this year's EVA conference. It consists of three sections, each written by one of the panellists and presenting a view on picturings and representation. The first section, by Annamaria Carusi, sets the context of the debate by outlining the main points of Scruton's argument, and raising three points of criticism of that argument, from the perspective of philosophy of art and of technology; the second section, by Gordana Novakovic, broaches the questions from the perspective of practicing artist, and raises further criticisms relating to the nature of digital interactive art, and the interface between neuroscience and art; the third section, by Timothy Webmoor, raises a set of questions from the perspective of science and technology studies.

The three views outlined raise many overlapping issues. They converge on some points, and diverge on others. We did not attempt to arrive at agreement with each other prior to the panel discussion, since we are aiming first and foremost, to make space for a variety of views and perspectives. This paper's further development, therefore, must be postponed until after the panel discussion. 


\section{A VIEW FROM PHILOSOPHY OF ART AND TECHNOLOGY: ANNAMARIA CARUSI}

In an article which is now a classic in the philosophy of art, a status it acquired in virtue of how much discussion and disagreement it has provoked rather than in the view that it expressed a lasting and deep truth about the nature of photography, the philosopher Roger Scruton claimed that photographs are not representational art. A photograph may be a representation in the same way as a realist or representational painting in that it shares (in some respects) the appearance of its subject; but a photograph's mode of sharing this appearance is not, for Scruton, one that can hold our attention or interest, or finally have the same kind of aesthetic value, as the way in which a painting comes to be a representation. The central reason for this is the means through which each comes to have a certain appearance: a photograph has a particular visual appearance because of a causal relation between the device and the subject photographed; a painting, instead, has a particular visual appearance because of an intentional relation between the artist and the subject depicted. In this paper, we shall not focus so much on the question whether in fact Scruton was right in classifying photographs and paintings correctly on the side of the causal and the intentional. Rather we focus on the causal/intentional distinction itself as a way of understanding why representations hold our interest, and why they are of value to us.

A causal relation implies that particular inferences can be made about the existence or nature of the subject represented: for example, from a photograph we are able to infer that some particular thing was actually there to be photographed and looked at in a particular way at the exact instant of being photograph being taken. The 'thereness' of photographs have been commented on by several others, including Roland Barthes, Susan Sontag and many others. We can be mistaken about the identity of the subject in the photograph, and we can be mistaken about what the particular thing actually is (a topic of great interest to science imaging) but that there is a something that appears a certain way - of this we can be certain (in the absence of meddling and manipulation - but this is too large a topic to be discussed here). For painting, instead, no particular thing need exist at a particular instant of time for the painting to have the visual appearance that it does. In order to answer the question why a painting looks a certain way, it makes sense to refer to the intentions of the painter; a painting is a representation by reference to the intentions of the artist (and here, we need to leave aside a deeper analysis of what exactly is meant by intentions, such as, the extent to which intentions are fully conscious or 'inner'. Scruton does not imply that they are either of these in this paper and these views are inconsistent with his more general philosophy of art. The terms 'intentions' and 'intentionality' in this paper cover intendings in the narrow sense (conscious intentions to carry out particular acts) and intentionality in the broad sense, of taking one thing to be about another, as occurs in perception, but also in meaning systems of all kinds). According to Scruton, a photograph is a representation as a record and not as the fulfilment of an intention, and that is also where its interest as a representation also lies. Of course photographs do involve intentions, including representational, stylistic, and aesthetic intentions, but these are not that in virtue of which they are representations - think of a CCTV camera. In a painting, instead, the artist presents us with a way of seeing the subject - and that's also where the interest lies. What I see in a picture is not only its visual properties, but an interpretation of this mode of seeing. The point of a painting of a person, for example, is not ' $x$ looked like that', but 'he was seen like that by the painter', and we are interpreting the mode of seeing as well as the visual appearance, or in the visual appearance. And this is precisely why paintings hold interest and value for us: because we're interested in modes of seeing, in interpretations, in the variety of ways things appear to ourselves and others and not just in the way they do appear to no-one in particular. Paintings - and all art - hold our attention because of this pivotal role of intentionality in them. Of course, photographs have intentionality too: photography involves numerous choices of angles, shots, lighting, choices and arrangements of subjects: but - according to Scruton - finally, the reason that they represent is a causal one which comes apart from all of these manifestations of intentionality. This is at the same time why codified representations, such as the codified ticker tape indication of a crystal's atomic structure (Scruton, 1981, p. 590), is for Scruton, not interesting as a representation: if we ask why it looks as it does, we need to look for the reasons in the code, rather than in a mode of seeing.

That intentionality has a central role in representation and the reasons we are interested in it is one claim. A further claim is that our interpretation of paintings is an interpretation of the ways in which intentionality is realised in a perceptual medium (in the case of painting a visual medium); and yet a further claim is that this involves us in interpreting or making sense of the 'characteristically human' (Scruton, 1981, p. 581).

Before we start to analyse terms such as intentionality, interpretation, and what, if anything, makes either of these 'characteristically human', let us recall that Scruton is not alone in holding the view that the mechanism whereby photographs 
come to be representations is not the reason for which we value them as representations. Scruton himself quotes James Joyce, in the Paris Notebooks of 1904:

Question: Can a photograph be a work of art? Answer: A photo- graph is a disposition of sensible matter and may be so disposed for an aesthetic end, but it is not a human disposition of sensible matter. Therefore it is not a work of art.

But many others have expressed a similar view. The expression of the view in the film Monster's Ball is as good as any other, and possibly more powerful. This exchange between Lawrence - the convicted murderer - and Hank - the prison guard -- occurs the night before Lawrence's execution, as he is drawing Hank's portrait:

HANK

My daddy took me to one of them picture men at the store, when I was little. Told me, when the man gave him the photo, they started to laugh. Said I'd never keep a woman with the face I got.

\section{LAWRENCE *}

I've always believed that a portrait captures a person much better than a photograph. Takes a human being to really see a human being.

It is this role of human seeing, seeing as, taking to be, sense-making and the whole range of intendings, and the way in which they define what it is for something to represent something, that is at the heart of Scruton's argument about representation, and the reason for which it is still worth returning to, even now when the discussion regarding whether photographs represent and have aesthetic value in virtue of their representing is rather antiquated. At the heart of this argument is the idea that nothing is a representation in the absence of the human interest in making it so; and that an important reason why we are so compellingly interested in aesthetic representations is what they tell us about human intentionality (in the broad sense!). The assiduousness with which we interpret works of art is an extension of our interest in other human doings and ways of being.

If Scruton makes a mistake regarding where, in this distinction, he places photography and paintings, it is still interesting to ask whether this distinction is one which we can use to explore the representational nature of digital and computational art and images of all kinds. In the rest of this section, I wish to highlight three points for discussion: the first is Scruton's understanding of the camera as a technological device which results in his placing it incorrectly in the causal/intentional divide; the second is the fact that digital and computational art complicate this distinction by bringing in a further term: information; the third is that Scruton's distinction in fact depends on a further distinction, that between representing and represented thing which is not as absolute as is assumed for the purposes of his argument regarding what does and what does not represent.

\subsection{Questioning the place of cameras and other technologies on the causal/intentional spectrum}

On the first point: Scruton assumes that the camera is purely causal and that it does not, itself, embody intentionality. However, as a device, the camera is an intentional device, in that it has its place in the ongoing history of humankind's inventiveness in finding ways of extending our own intentionality: that is, our own perceptual capacities. In this sense, the camera is not that different from the human visual system which has causal as well as intentional aspects: in order to see anything, we need to direct our attention to some things rather than others in our visual sphere, but we also have to have our eyes open so that the retina can be affected by light. It seems arbitrary to say that one causal/intentional mechanism results in a representation whereas another does not. Rather, the technologies that come to play a role in the way that we make sense of the world around us are themselves a way of playing out different modes of relating causality and intentionality, and even of modifying and extending them. Thus questions of representation cannot be considered independently of the technological systems that are used for representing. The technologies we use are not just a vehicle for achieving a representation, but are inextricably part of the act of representing and of our interest in representations and the value they hold for us.

\subsection{Questioning the causal/intentional distinction}

The second point is an extension of the previous point that technologies do not necessarily simply play out pre-existing modes of causality and intentionality but can modify them too. This is what we are seeing now with digital and computational imaging, which insert into the heart of the representational relation a third term, that of information. Software and computation generally are essentially ways of manipulating information. 'What is information?' is a complex question to answer. While it is not the same as a cause (it is not, for example, light itself but information about light that operates in digital imaging), it is also not straightforwardly the same thing as intentionality if we understand by that human intentionality. This is a different point from the frequently made point that that ultimately it is humans who program the 
computer and who therefore ultimately delimit the choices and decisions that form the representation). Rather the point is that the translations of light, contour, depth, etc, into information must also 'mean' something for the computer in order for the manipulations of information to be carried out, and that the process that ensues is not one that is fully due to human intentionality or physical causality, but to this further category of information acting upon information. Section 3.3 expands on this point as only someone like Gordana, able to experience the process from the inside, can do. This is the point where this form of representation breaks with the idea that representations are of such compelling interest to us because of what they tell us about human intentionality; or perhaps the reason they are so interesting is in virtue of the ways that the ways they disturb our conception of human intentionality? This is a point of speculation and debate which is taken up again in Section 4.

\subsection{Questioning the distinction between representing subjects and represented objects}

Finally, the third point is that there is a further distinction upon which the distinction between causal and intentional relations between visual artefacts and whatever is their subject is grounded. This relation could not be made without an underlying distinction which has on one side the representing item (painting, picture, diagram, image, visualisation, etc.) and on the other the represented item. In the case of visual more-or-less realist representation, this is often seen as the representing item and the represented item in some sense sharing the same appearance - as was seen in the case of Scruton. This is rather loose and vague - and rightly so - since there is an infinite variety of ways in which one thing can be said to 'look like' another or share its appearance. Here, I wish to focus on the idea that these two items can be so distinguished, or even that it is useful to do so in order to understand representation. Instead, I wish to point towards an alternate view of art and its meaning and significance to us. According to Merleau-Ponty, art expresses a connectedness and reciprocity between ourselves and the things with which we interact in our environments. Klee reported on the experience, when walking through a forest, of being looked at by trees as much of he looking at them, and Merleau-Ponty (1993, p. 127) expands on this point writing 'The eye [...] is that which has been moved by some impact of the world, which it then restores to the visible through the traces of a hand.' The important thing here is not the traces of the hand result in a 'copy' (however abstracted, inexact, skewed or faithful) of the visible; but that it expresses the ways in which the visible has an 'internal equivalent' in the perceiving (feeling, sense-making) body:

Things have an internal equivalent in me; they arouse in me a carnal formula of their presence. Why shouldn't these correspondences in turn give rise to some tracing rendered visible again, in which the eyes of others could find an underlying motif to sustain their inspection of the world? (Merleau-Ponty, 1993, p. 126)

In notes posthumously published, Merleau-Ponty suggests that the relation between perceivers and the world is not one whereby one is set over against the other, one on one side, and one on the other of some invisible divide, but rather that perceivers and world are in a circuit (2003: 223). Starting from one, we can't but get to the other, without being able to say when exactly we crossed over. Art then is a way of expressing these crossings over between ourselves and the worlds we inhabit. The only thing that I would want to add is that this circuit includes the technologies that we use for the 'crossings over' - in ways that we have yet to explore.

In this section we began by considering Scruton's distinction between photography and painting on the basis of the causal or intentional ways in which they come to be representations and the implications he believes this have for the interest that paintings and photographs can have for us human viewers - as representations. We then went on to question the way in which technologies are not considered by Scruton as themselves playing out causal/intentional relations, and even of making available different ways of thinking and experiencing these ways of being related to the world around us; we also questioned whether this distinction is sufficient to account for representations, and proposed that digital and computational technologies in fact bring into play another relation, the informational one. But this type of relation, actualised in computers, raises questions about human intentionality as the focus of interest in representations. Finally we have questioned the underlying distinction between representing and represented items, and proposed - following Merleau-Ponty - that there is not such a clear distinction to be made, but that rather, the artefacts that we produce for aesthetic interest may be ways of expressing a relations of reciprocity and crossing over between human beings and the world they inhabit, in which technologies play a crucial role.

\section{A VIEW FROM THE ART/SCIENCE INTERFACE: GORDANA NOVAKOVIC}

My exploration of the questions put to the panel is undertaken from the point of view of a practising 
artist who uses digital technologies to produce collaborative interactive artworks that are inspired and informed by science. The easiest way for me to structure my answers is to change the key question slightly by replacing 'picturings' with 'interactive digital artworks', and then to use some of Roger Scruton's observations on art, photography and cinema as points of contrast. This is not intended as a way of criticising Scruton's views, which were expressed before digital technologies reached the mainstream, but as a way of bringing into the foreground his assumptions about the artist, the subject/object of representation, the artwork, and the viewer/participant, all of which are violated by the current practice of many artists, including myself. My approach to the question will be to examine these assumptions, and perhaps to show how the results of this examination can themselves constitute an answer.

\subsection{The artist}

Scruton's paradigm case (his 'ideal painting') is of the solitary artist producing a work that represents some inner vision; it is the individual artist's intention that separates painting from 'ideal' photography, which is merely causal. This emphasis on the individual is carried over to his analysis of cinema, where he attributes the intention behind a film to the director. However, in the case of film, it is certainly not the case that the final version of what may legitimately regarded as an artwork is the product solely of the director's intention. Making a film is a collaborative enterprise where many roles and professions other than the director's also contribute to the artistic dimension for example, the actors, scriptwriters, and cameramen, among others - and the eventual output is constrained by material and financial factors that often lead to the final version falling short of the director's original vision (hence the phenomenon of 'the director's cut'). I am not sure how easy it would be to extend Scruton's arguments to include this messy reality in which there is no longer any single intention that the artwork can represent.

Some art, at least, has moved on from the model of an object being wholly produced by an individual. This is particularly true of art, such as digital art, that depends on technologies that demand specialised knowledge that the artist does not possess. For example, much of my work over the last couple of decades has involved computer programmers, electronics engineers, and artificial intelligence experts. However, in my approach, these specialists are not merely the necessary tools for bringing my vision to reality; they and I form a collaborative team of equals, and the eventual output is ours, not mine. This idea of the team can also be seen in cinema, where some well-known directors sometimes abandon the idea of individual credit, as in Godard's 'Bande à part', or 'Nouvelle Vague', or structure the development of the film as the outcome of ongoing workshops with the (sometimes amateur) actors, as in Peter Watkins' 'La Commune'.

Digital technologies have also enabled and facilitated the use of generative techniques, where the role of the artist(s) and her/their collaborators is essentially to define a visual or other space that the artwork itself will explore. The detailed output of the final piece (which may or may not be time-based) is not completely known before it is produced, and in the case of the use of random or chaotic techniques may not even be knowable in principle. Exactly what is intentional here, where the outcome may go beyond anything conceived by the artist?

\subsection{The subject/object of representation}

The proliferation of new technologies has made it possible to access detailed information about things, typically involving very large (cosmic) or very small (subatomic) scales, that were previously confined to the realm of theoretical speculation. This has given rise to an entirely new field dealing with the representation of these (and other) scientific findings, and it has also become a source of inspiration for artists, particularly for the new discipline of art science.

For 'scientific' visualisations, sonifications and audio-visualisations, there is of course a very natural concern with objectivity. In his essay, Roger Scruton discusses the relationship between the object of representation and the representation itself, especially in Section 6 where he considers his idea of representation in the context of his 'ideal photograph', and touches on the issue of the representation of scientific data with the example of an electron microscope which 'punches out on a ticker tape a codified indication of a crystal's atomic structure'. He asks whether this is a representation of the atomic structure, and decides that it is not because 'such a concept of representation would be uninteresting indeed'. Here he misses two opportunities. The first is to point out, as any scientist would, that the atomic structure is not itself an absolute fact, but a theory expressed in the form of a model. There is no reality to represent - there is only an interpretation of the data that may turn out to be mistaken, just as Newton's interpretation of his data had to give way to Einstein's. The second is to observe that scientists rely to a great extent on visualisation - open any physics, chemistry, or biology textbook to see this - and the really interesting question for science is: what aspects of a visualisation make it a good representation of a theory that is itself an interpretation of data? 
Within the realm of representing or using scientific data or models in art, the question of truthfulness seems to be inappropriate as long as the underlying scientific data are accurate. We might even say that all non-distorting representations of scientifically valid data are equally valid representations of natural phenomena. However, the real difference between an artistic interpretation and a representation produced specifically for scientific purposes is in the motivation, which entails the use of different methods and different final outcomes. For artists, the scientific findings, data or theory are only the starting points in the search for a metaphor. The artist's intention is not limited to representing natural phenomena, but seeks to express ideas and/or emotions and to create conditions for communicating their vision to others. Of course, there is an endless variety of ways that this can be done. And if there is in the end a striking resemblance to the relevant natural phenomena, or a meticulous integration of accurate scientific findings within the artwork itself (in whatever form it might take), all of that is in the service of an artistic concept, and is not a primary motivation.

\subsection{The artwork}

Scruton's view of an artwork (including a film) is essentially of a passive and unchanging object, image, or set of images. An interactive digital artwork is none of these - and neither is our daily life in the $21^{\text {st }}$ century. The moment we step from the world of objects into the digitally enabled and facilitated world - whether in life or in art - this step resembles in a way the leap from classical to relativistic or quantum physics. Digital technologies have brought unprecedented changes, transforming all aspects of human activities in a very short period of time. As Virilio observes, 'We are no longer in the world of Newton, but in the world of Einstein' (Virilio, 2001). Everything appears familiar, but operates according to different laws, appears as ambiguous, and does not follow the familiar cause-and-effect linearity. In this context, the static object seems no longer to be adequate for manifesting artistic ideas.

Tightly linked to, and enabled by, rapid technological development, new media and electronic arts have changed contemporary art with the introduction of interactivity, and with a constant emergence of new art forms and categories. Instead of Scruton's paintings, photographs, sculptures, or prints, digital technologies offer a broad and ever-increasing selection of interfaces. Technological platforms range from mobile phones, through powerful laptops, to large networks controlling multiple lasers and light-beams projecting onto the sky; new ones appear almost on a daily basis, and any list will soon be out of date. The available artistic media now range from traditional localised and individual fine art techniques, to crowd sourcing on the distributed World Wide Web. However, they all have one thing in common: they are designed to transform and present digitally processed, generated or manipulated data into forms that our senses can engage with. Critically, the choice of forms and modalities is to a great extent free and flexible; a single computational process applied to the same set of data can now be presented by means of a huge variety of interfaces, dramatically changing the final outcomes and experiences.

In relation to Roger Scruton's article, how might we discuss intentionality and causality in the context of digitally enabled arts? In the realm of digitally enabled artefacts, the active nature of the software and hardware becomes significant. Hardware and software are not just tools any more. The computational processes involved often operate to a large extent beyond human control, and beyond the human level of intervention. In the most complex form of interactivity, the artefact itself changes as a consequence of interaction; equipped with its own 'nervous system', it 'senses' the participant, processes the received data and reacts accordingly, often using quasi-organic computer programmes. In The Address of the Eye: $A$ Phenomenology of Film Experience, Vivian Sobchack offers a brilliant phenomenological analysis of cinematography, and of intentionality, and in particular of 'machine-intentionality', in film (Sobchack, 1991). Could we similarly attribute some form of extended 'machine-intentionality' to interactive installations? This goes far beyond Scruton's idea of an artwork as a fixed passive object.

We can take this further. An interactive installation can be described as a computer controlled and facilitated information exchange between two entities: the installation, and the participant. Creating an interactive work involves defining the software and hardware architecture that determines, provides and supports the interaction between the two entities. Because our sense of self depends critically on the perceived boundary imposed by the body, which is in turn mediated by the processing of sensory information, the intervention of the installation in this process can dissolve or alter this boundary. This means that clear boundaries cannot be defined either for the participant's bodily sense of self, or for the installation, and this makes it impossible to describe the installation as an object. Where can we go from here?

In fact, interactive art is best defined as a process, or, more specifically, a complex system of 
interdependent processes. In contrast to Scruton's fine art artefacts, an interactive installation is not a passive reflective object. It creates a dynamic feedback process between the human body and the system. It is the participant who sets the interaction in motion and induces the process of interaction that depends upon h/er presence and activity. On the other hand, an interactive installation is dynamic and emissive, and tends to overpower the human body and mind; through the interaction, the participant can become an almost passive receiver. It is then the case that the installation actively manipulates our perceptions, rather than that we make sense of the perceptual situation. Compared to both cinematographic forms and fine art objects, the dominance of the role of the installation in this perceptual situation could be said to put the participant in the position of replacing the perception of an object by the observation of perception itself.

\subsection{The viewer/participant}

In his essay, Scruton says little about the viewer, but implies that s/he is a passive spectator in whom a certain experience will reliably be induced by the artwork. By making reference to the art of previous centuries, there is an implicit assumption that time and culture do not matter, or do not matter much. While this may be true to some extent in the context of painting, it is certainly false in the context of digitally enabled interactive art: established perceptual habit and familiarity play a significant role in our experience of digital media, and therefore in our response to interactivity. We know that our responses to sensory stimulation are shaped by our previous exposure to the standardised forms presented on TV or computer screens, and to our long-term exposure to typical audiovisual rhythms. This can lead to reduced perceptual capacity - for example, the auditory bandwidth of a citizen of a large Western city is much smaller than that of one who spent all her life in an intact natural environment.

It can also lead to cognitive effects, and this led Peter Watkins, in his analysis of the audiovisual mass-media industry, to coin the term Monoform (Watkins, 2007). The Monoform is a standardised audiovisual language marked by a fragmented, high-speed succession of diverse sensations that fracture attention, and suspend reflective processes. But, as Watkins notes, '...a consistent use of the Monoform - with its total absence of time for reflection, its apparently seamless (and thus unquestionable) narrative thrust, its constant monolinear direction forward (denying flexibility of memory, and complexity of human experience) has had both obvious and incalculable long-term effects on our feelings. It has desensitised us to many of the things that occur both on the screen, and everywhere around us...' The response to an interactive installation of an individual who has been exposed to the Monoform for decades or more will be very different from that of someone from a less technologically developed culture.

There are large differences even between individuals within the same culture. It might help to give an example from my own practice in connection with the ongoing Fugue project (Novakovic, 2009). In Fugue, the virtual immune system engages with a fictional virus. At the heart of the piece is a complex piece of scientific software, an artificial immune system algorithm, which accurately mimics the cascading responses to infection of the human immune system. No two responses will ever be the same, and the time scale and outcome of the struggle is always unpredictable. However, Fugue does not just display what could be seen under a microscope. Instead, we have transformed the data generated by the artificial immune system into symbols that express the dynamics and the rhythm of the biological processes. While the visuals reflect one view of events in the underlying immune system when it is infected by an artificial virus, the sound reflects another, and always in a new and unpredictable way. The piece has been exhibited in various formats, using different interfaces, but in all of these the computational processes remained exactly the same, except in the case of the full interactive version where an additional software package enabled the system to respond to the participants as if they were yet another component of the computational model of the immune system.

In spite of the fact that in all its variants the underlying software is identical, the responses from the participants have been quite unpredictable, and they have varied largely depending on the form of the interface. In the simplest screen-based free running display, a significant number reported an immersive, almost meditative experience, although no classical immersive technology was involved. Even the author of the 3D modelling, Dr Anthony Ruto, remarked that he sometimes plays it on his computer, in his own words, 'just to relax'. This response contradicted our expectations, especially since we had designed a full-scale interactive installation with the intention of inducing meditative effects, but none of the participants had reported any. However, different age groups responded in very different ways to the interactive installation. Those who had grown up with digital technologies, the so-called 'digital natives' (Prensky, 2001), put considerable effort into trying to uncover the triggers for the interactive effects. (They were unsuccessful because this was, in fact, deliberately made impossible.) Their behaviour contrasted sharply with that of the non-natives, namely the generations who had encountered digital and 
interactive technologies later in their lives, who enjoyed exploring and experiencing the environment in a playful, relaxed way, without consciously attempting to manipulate it. It appears that both habit and familiarity with technology play significant roles in the ways that people perceive and react to digital images and sounds, and that these factors may become most transparent in interactivity.

It seems clear that the theory and practice related to traditional object-oriented plastic arts are of little help in understanding the phenomena associated with interactive digital art. However, the nature of the complex processes involved when the human body and mind encounter and interact with digital technologies has now become a focus of attention in various fields. Within the sphere of digitally mediated art, researchers and practitioners have explored many of the technical, conceptual, and experiential aspects of this enigma over the last forty years, but one critical aspect - exploring and exploiting the changes in the brain that are directly related to the nature of digital technologies - is still relatively untouched.

A potentially promising approach is the neuroscientific subdiscipline of neuroaesthetics, pioneered by Professor Semir Zeki (1999). This groundbreaking paradigm has opened an entirely new area in which both artists and neuroscientists look at the neurobiological basis of creating and experiencing the plastic arts. Working within the scientific concept of the visual brain, it is essentially focused on vision and static objects. However, the area of contemporary artistic practice that neuroaesthetics leaves unexplored are the multisensory experiences within the growing body of process-based arts enabled by digital technologies, such as interactive art.

A possible new direction has recently appeared with the emergence of the science of brain plasticity, the study of the ways in which the brain can radically reconfigure itself under certain conditions. This has conclusively shown that the brain can no longer be regarded as a fixed, closed, passive receiver of information from the senses $-a$ mere processor for the information that is controlling our body through a kind of one-way communication. We are now seeing the recognition of growing scientific evidence that the brain is in fact almost nakedly open to external influences, and is capable of rapid and radical change by remodelling itself through learning and interaction with the environment. What is needed now is for these insights to be extended and explored in the context of art, perhaps in the ways outlined in my Manifesto for Neuroplastic Arts (Novakovic, 2007).
But will neuroscience bring the final answers to all perception-related questions, the enigma of digitally enabled artefacts included? Perhaps not, because a new and strong critique that challenges many current dogmas has now appeared from the field of neurophenomenology, a discipline firmly grounded in the philosophy of Maurice Merleau-Ponty, but also embracing recent research in neuroscience. A key figure is Alva Noë, who is part philosopher, part cognitive scientist, and part neuroscientist. Together with Evan Thompson and others, he offers a hypothesis about perception in action which builds on Merleau-Ponty's idea of perception as a process of interaction between the embodied and situated human and the world. ( $\mathrm{He}$ also believes that artists should not just be objects of scientific investigation, as they are in neuroaesthetics, but should actively contribute to the fields of perception and consciousness studies.)

Noë's iconoclastic views can be seen in his most recent book Out of our Heads (Noë, 2009) where he says: 'Our culture is obsessed with the brainhow it perceives; how it remembers; how it determines our intelligence, our morality, our likes and our dislikes. It is widely believed that consciousness itself, that Holy Grail of science and philosophy, will soon be given a neural explanation. And yet, after decades of research, only one proposition about how the brain makes us conscious-how it gives rise to sensation, feeling, and subjectivity-has emerged unchallenged: we don't have a clue.' It will be interesting to see whether and how Noë's approach will affect our understanding of interactive digital artworks in the future.

\subsection{Coda}

Although I have not answered the original question directly, I hope that I have shown that, in my own practice, the questions that most need answering are those hidden in the assumptions made in the paper that is the focus of this panel.

\section{A VIEW FROM SCIENCE AND TECHNOLOGY STUDIES: TIMOTHY WEBMOOR}

Shifting Scruton's question to the register of digital modes of engagement is helpful as long as we recover his starting position in metaphysical speculation, rather than build from or contest his conclusions concerning representation. That is, his conclusions for aesthetic value clearly rest upon a separation of human agency from what might be termed mechanical or nonhuman agency. Nevertheless, his initial invitation to speculate about the nature of agency in terms of representational practices is both useful and potentially heterodox. 


\subsection{Science, representation and ratios of agency}

Philosophers and historians of science have similarly developed criteria for representations through underscoring the ratios of humannonhuman agency involved in their production. Daston and Galison's (2007, p. 115) epistemic virtue of 'mechanical objectivity,' dominant in the late $19^{\text {th }}$ century, emphasises the 'causal' type of relationship Scruton attributed to the celluloid chemistry of the camera and the ambient light rays entering the aperture. The increasing ubiquity and affordability of instruments for recording during this threshold period of early modern science encouraged a technological 'hype.' Ostensibly about the instruments to aid the 'scientific imagination' (Hankins and Silverman, 1995), the technophilia was really about the representational prowess they could bring to scientific research. Such subject-less image producing representations facilitated the expansion of experimental science. Physically, through requiring more laboratory space, technicians, infrastructural and institutional support for the sophisticated equipment. But, more importantly, the greater production of these mechanically standardised representations allowed them to be circulated widely amongst far-flung colleagues without distortion. As Latour's (1986) 'immutable mobiles' these instrumental inscriptions functioned as epistemic circuitry enervating an increasingly global science. A physical network of circulating representational results that worked as an 'epistemological engine,' accelerating scientific advances through expanding practitioners' ability to 'virtually witness' experiments at-a-distance (Woolgar \& Coopmans, 2006; Shapin, 1984). The trust to accept, build upon and develop the experimental results of others based upon the collateral security of representations rendered through the neutral and uncaring causality of technology.

\subsection{Machinic agency}

It seems that, according to Daston and Galison's reconstruction, early modern science thought good representation was the absence of human agency. Such machinic objectivity gave great ballast to the progress of early modern science. It is no wonder, then, that the 'enchantment of technology' (Gell, 1992) continues to hold many spellbound to the epistemological promises of representations produced by instruments in the service of science. Of course, work by scholars of contemporary science (Daston \& Galison, 2007; Hacking, 1983), particularly those examining everyday practices of scientists (Amann \& Knorr-Cetina, 1990; Latour \& Woolgar, 1986; Latour, 1986), underscores that instruments do not produce representations alone. Contrary to Scruton's portrayal of a purely causal camera, making scientific representations involves practitioner and technology in a collective activity more akin to craftwork; a material engineering of media. So while Scruton's causal camera and the 'mechanical objectivity' of early modern science described by Daston and Galison use inverted ratios of human-nonhuman agency for defining 'representation,' they both uphold this fundamental division that may no longer be so analytically useful for understanding representations.

\subsection{Collective agency}

What if, as Daston and Galison (2007, pp. 39-41) intimate and many Science and Technology Studies scholars explicitly suggest (e.g. Latour, 1992), we think through representation not in terms of the repetitive debates of epistemology, but rather ontologically? To go back to Scruton's initial speculation in terms of agency, yet not insist upon classifying agency according to what is human and what is machinic. That is, to not separate off representation from 'representer.' What would representation look like under an agnostic agency? There are other motivations for doing so, particularly with respect to digital images.

Firstly, as Anne Freedberg (2006) and other art historians admirably detail, unlike Leon Battista Alberti's initial realist windows, digital images as 'virtual windows' are dynamic interfaces. More interactive display-cum-control panels than the isolated information of analog representations. As digital images become ubiquitous, attached to us as 'everyware,' haptically responsive and increasingly visually immersive with gaming and social networking 'second worlds,' the phenomenological lesson of relatedness becomes technologically embodied. Already our mode of everyday engagement with digital images - on our iPhones or before our many Graphical User Interfaces - reminds us of our cyborg nature. These so many visual prostheses augmenting 'our' agency (Manovich, 2006).

Secondly, if we consider digital images from an ontological perspective, we sidestep the many contentious and irresolvable disputes that bind 'representation' to epistemological worries of fidelity and correspondence (Putnam, 1988). These debates would continue to have us, as they did for early modern science, making arbitrary incisions between proverbial objects and subjects. Now, these long-standing divisions were thought to help ascertain whether representations were produced according to the Mertonian ideals of science that tipped the ratio of agency away from subjectivity. With digital media, we must still sift out signal from noise in our images. But thinking in terms of a collective agency shifts their evaluation as 'representations' to their ontological capacity for 
action. It is in keeping with an engineering and design sensibility. What matters is their effect or their capacity for action in performing tasks.

Our collective work with digital images amounts to Hacking's (1983) electrons and positrons. Along with other phenomena in particle or astro-physics, we may endlessly debate their reality in terms of how they are represented; whether this or that image is an accurate and undistorted proof. But the collective action of practitioners and instruments allows them to be sprayed onto a niobium ball to alter charges. Their efficacy is real so they are not doubted. That is, in step with the increased sophistication of instrumentation, our collective capacity for 'intervention' comes to be the epistemic guarantor of scientific results: 'It is because entities that in principle cannot be 'observed' are regularly manipulated to produce a new phenomenon and to investigate other aspects of nature' (Hacking, 1983, pp. 262). Fidelity may be futile, whereas pragmatic criteria, the ability to get work done and accomplish specific tasks in research, are what count.

Thinking in terms of the collective agency of ourselves-with-our-media may indeed seem speculative and heterodox. It runs contrary to Scruton's and most other anthropocentric approaches to agency. Or rather, it gives up on splitting hairs over attributing causality through the determination of agency's owner as if it were a proprietary commodity. It is an approach more like digital 'copyleft.' Use is encouraged as opposed to ownership. Moreover, in emphasising output or effect, a collective agency inverts a search for 'origins' or the initiation of action, as with Scruton's human endowed with Franz Brentano's intentionality. Agency comes as the end, not the beginning, of representation. It is performed, not a background condition.

So, are digital picturings or images representations? If we remain insistent with Scruton that human intention is the magic ingredient of representation, then we can say yes. Or at least that they are 'part representation.' On the other hand, if we obdurately demand with $19^{\text {th }}$ century scientists that representations must be subjectless, we can equally affirm their status as part representations. Perhaps more usefully, and in step with our increasing immersion in digital media, is to suggest that we are part of any complete representations.

\section{CONCLUSION}

There will no doubt be more than these three views and perspectives at the panel discussion, and in the broader discussion around these issues. Rather than trying to conclude neatly on a common view that we can all share - which, just because it can be universally shared, will probably not be the most interesting insight about the potentiality of digital picturings - perhaps we can only point to a common attitude, and that is, that it makes a difference how we conceive of these digital picturings that are currently forcefully shaping our visual world. Despite drawing upon different literatures, fields, and practices, there is a convergence in the three perspectives put forward here on the view that the inherited categories for understanding representation, with their attendant baggage of dichotomies, between human and machine, between subject and object, and between represented and represented thing, are no longer tenable. Force for abandoning these distinctions comes from the very topic of this conference and from our modes of engaging digital media.

\section{REFERENCES}

Addica, M. and Rokos, W. (2001) The Monster's Ball. The daily script. http://www.dailyscript.com/movie.html (8 April 2010).

Amann, K. and K. Knorr Cetina. (1990) The Fixation of (Visual) Evidence. In M. Lynch and S. Woolgar (eds), Representation in Scientific Practice. The MIT Press, Cambridge, Massachusetts.

Daston, L. and P. Galison. (2007) Objectivity. Zone Books, New York.

Freidberg, A. (2006) The Virtual Window: from Alberti to Microsoft. The MIT Press, Cambridge, Massachusetts.

Gell, A. (1992) The Technology of Enchantment and the Enchantment of Technology. In J. Coote and A. Shelton. (eds), Anthropology, Art and Aesthetics. Clarendon Press, Oxford.

Hacking, I. (1983) Representing and Intervening. Cambridge University Press, Cambridge.

Hankins, T. and R. Silverman. (1995) Instruments and the Imagination. Princeton University Press, Princeton.

Latour, B. (1986) Visualization and Cognition. Thinking With Eyes and Hands. In H. Kuklick and E. Long (eds), Knowledge and Society: Studies in the Sociology of Culture Past and Present. JAI Press, Greenwich, Connecticut and London. 
Latour, B. (1992) We Have Never Been Modern. Harvard University Press, Cambridge, Massachusetts.

Latour, B. and Woolgar, S. (1986) Laboratory Life: the Construction of Scientific Facts, $2^{\text {nd }}$ edition. Princeton University Press, Princeton.

Manovich, L. (2006) Visual Technologies as Cognitive Prostheses: A Short History of the Externalization of the Mind. In M. Smith and J. Morra (eds), The Prosthetic Impulse: From a Posthuman to a Biocultural Future. The MIT Press, Cambridge, Massachusetts.

Merleau-Ponty, M. (1993) Eye and Mind. In Johnson and Smith (eds), The Merleau-Ponty Aesthetics Reader: Philosophy and Painting. Northwestern University Press, Evanston.

Merleau-Ponty, M. and Séglard, D. (2003) Nature: Course Notes from the Collège de France. Nortwestern University Press, Evanston.

Noë, A. (2009) Out of Our Heads: Why You Are Not Your Brain, and Other Lessons from the Biology of Consciousness. Hill and Wang.

Novakovic, G. (2007) Metropolis: An Extreme and Hostile Environment. http://mutamorphosis.wordpress.com/2009/01/15/m etropolis-an-extreme-and-hostile-environment/ (4 April 2010).
Novakovic, G. (2009) Fugue - Art and Science Collaboration. http://www.fugueart.com

(4 April 2010).

Prensky, M. (2001) Digital Natives, Digital Immigrants. From On the Horizon, MCB University Press, Vol. 9 No. 5.

Putnam, H. (1988) Representation and Reality. The MIT Press, Cambridge, Massachusetts.

Scruton, R. (1981) Photography and

Representation. Critical Inquiry, 7, pp. 577-603.

Shapin, S. (1984) Pump and Circumstance: Robert Boyle's Literary Technology. Social Studies of Science, 14, pp. 481-520.

Sobchack, V. (1991).The Address of the Eye: A Phenomenology of Film Experience. Princeton University Press.

Virilio, P. (2001) In J. Armitage (ed) Virilio Live: Selected Interviews. Sage, London. p. 83.

Watkins, P. (2007) Five Years Later - The Media Crisis. http://pwatkins.mnsi.net/Intro MedCr.htm (4 April 2010).

Woolgar, S. and C. Coopmans. (2006) Virtual Witnessing In a Virtual Age: a Prospectus for Social Studies of e-Science. In C. Hine (ed), New Infrastructures for Knowledge Production: Understanding e-Science. Information Science Publishing, London. 